porting the unnecessary use of motorised vehicles. We shall give priority to ecologically sound means of transport (in particular walking, cycling, public transport) and make a combination of these means the centre of our planning efforts. Motorised individual means of urban transport ought to have the subsidiary function of facilitating assess to local services and maintaining the economic activity of the city.

\section{Responsibility for the Global Climate}

We, cities \& towns, understand that the significant risks posed by global warming to the natural and built environments and to future human generations require a response sufficient to stabilize and then to reduce emissions of greenhouse gases into the atmosphere as soon as possible. It is equally important to protect global bi- omass resources, such as forests and phytoplankton, which play an essential role in the earth's carbon cycle. The abatement of fossil fuel emissions will require policies and initiatives based on a thorough understanding of the alternatives and of the urban environment as an energy system. The only sustainable alternatives are renewable energy sources. [...]

\title{
Sustainability-Ansätze vor Ort Die Ulmer Nachhaltigkeitsinitiative
}

\section{Was bedeutet regionale Nachhaltigkeit?}

Im Bericht der Brundtlandkommission wurde nachhaltige Entwicklung definiert als die Forderung an die heutige Generation, so zu leben, daß die Bedürfnisse der zukünftigen Generationen nicht beschnitten werden. Es ist wichtig, daß solche Einsichten in weltweiten Foren behandelt werden. Die Rio-Konferenz war ein notwendiges und wichtiges Ereignis. Doch wir wissen auch, daß es sehr schwer ist, daraus zu spürbaren Änderungen zu kommen.

Spürbare Änderungen lassen sich aber auf regionaler Ebene verwirklichen. Lebensstile und auch Wirtschaftsweisen sind stark regional geprägt. Die Region ist überschaubar. Die Akteure sind bekannt. In der Region können Ursachen und Wirkungen von Handlungen zugeordnet werden. Verantwortung ist zurechenbar. Umweltkatastrophen und Unfälle erzeugen unmittelbare Betroffenheit. Diese Betroffenheit hat Folgen, denn sie kann in konkrete Handlungsmöglichkeiten münden, die für den konkreten Alltag gelten.

Die o.g. Definition ist für regionale nachhaltige Entwicklung ungeeignet. Ich verstehe darurter Folgendes: Regionale nachhaltige Entwicklung ist ein Leitbild, das in einem langfristigen Suchund Abstimmungsprozeß erreicht werden soll. Dabei sind die gesellschaftlichen Nutzungsansprüche in Übereinstimmung zu bringen mit den natürlichen Lebensgrundlagen. Aus diesem Vergleich ergeben sich Nachhaltigkeitslücken, die unter der Zielsetzung von intertemporaler Gerechtigkeit verkleinert und beseitigt werden sollen.

\section{Regional nachhaltige Entwicklung}

Der Ansatz regionaler nachhaltiger Entwicklung geht davon aus, in möglichst vielen Bereichen und Sektoren solche Nachhaltigkeitslücken zu ermitteln und konkrete Handlungsmöglichkeiten aufzuzeigen, die diese Lücken verkleinern und beseitigen können. Dabei müssen einige wichtige Bedingungen beachtet werden:

- Die erste Bedingung lautet, daß die Region eingebettet ist in eine Vielzahl von Regionen, mit denen Lieferbeziehungen bestehen: Ex- port und Import von Rohstoffen, Energie und Schadstoffen, die auch in Produkten enthalten sind.

- Die zweite Bedingung lautet, daß unterschiedliche gesellschaftliche und politische Handlungsebenen $\mathrm{zu}$ beachten sind: Das Land Baden- Württemberg, die Ebene der Bundesrepublik Deutschland, die europäische Ebene und die globale Ebene (UN).

- Die dritte Bedingung für den Such- und Abstimmungsprozeß lautet, daß demokratische Strukturen vorherrschen mit freiem Meinungs- und Informationsaustausch.

Schließlich muß noch beachtet werden, daß nachhaltige Entwicklung ein Phänomen darstellt, das ökonomische, ökologische und soziale Dimensionen aufweist (Naret).

\section{Strategien für regionale Nachhaltigkeit}

\section{Voraussetzungen}

Der Ulmer Initiativkreis nachhaltige Wirtschaftsentwicklung e. V. geht davon aus, daß ein Anstoß zu nachhaltigem Strukturwandel möglich ist, wenn (nach Manfred Linz)

- das zu verfolgende Projekt Sinn macht;

- ein ehrlicher Makler die Koordinierung übernimmt,

- die Anstöße zur Verhaltensänderung alle beteiligten Akteure gerecht treffen,

- mit den Anstößen das konkrete Alltagshandeln verändert werden kann.

\section{Nachhaltige Wirtschaftsentwicklung macht Sinn}

Wir können davon ausgehen, daß die ökologische Restriktion den meisten bekannt ist. Sie löst jedoch in ihrer allgemeinen Beschreibung weitgehend folgenlose Betroffenheit aus. Wissenschaftlich fundierte Informationen über regionale Nachhaltigkeitslücken in bestimmten Sektoren und Bereichen sind wichtig. Dabei müssen sowohl Schwächen als auch Stärken und Potentiale der Region aufgezeigt werden. Es sollte deutlich gemacht werden, daß nachhaltige Wirtschaftsweisen und Lebensstile nur langfristig erreicht werden können, daß aber der Kurswechsel heute beginnen sollte, wenn große Anpassungsfriktionen und Kosten ver- mieden werden sollen. Es geht nicht darum, morgen eine Wende vollziehen zu müssen.

\section{Ehrlicher Makler}

Dem Ulmer Initiativkreis kommt die Rolle eines Mediators zu. Der Mediator sollte aus einer privaten Initiative entstehen, die nicht bestimmten gesellschaftlichen Interessen (Lagern) zugeordnet werden kann. Günstig ist eine überparteiliche Struktur der Mitglieder und eine wissenschaftliche Fundierung. Beides ist beim unw gegeben

Die Initiative ist durch Einzelpersönlichkeiten entstanden, die keinem Lager zugeordnet werden können. Der Vorstand des unw setzt sich aus zwei Wissenschaftlern und zwei Unternehmern zusammen. Die Parteiaffinität der Mitglieder streut über alle demokratischen Parteien. Die Arbeit des unw wird begleitet von einem interdisziplinär zusammengesetzten Wissenschaftlichen Beirat international renommierter Forscher, die Hälfte davon ist in Ulm ansässig. Die Mitglieder kommen aus allen gesellschaftlichen Gruppen. Zur Zeit sind die Wissenschaftler und Wissenschaftlerinnen zahlenmäßig am stärksten. Die Ulmer Stadtverwaltung ist mit Führungskräften stark vertreten. Der UImer Oberbürgermeister ist Gründungsmitglied und Schirmherr des unw, eine wichtige Voraussetzung für die Verfolgung der Ziele. Als nächststärkste Gruppe zählen die Unternehmen, sehr aufgeschlossen und aktiv sind wichtige Banken; auch die IHK ist Mitglied des unw. Weitere Mitglieder kommen aus verschiedenen gesellschaftlichen Gruppen: Gewerkschaften, Kirchen, Handwerk. Die Heterogenität der Mitglieder begründet den Kontakt zu den Akteuren der Nachhaltigkeit. Der unw betreibt eine Forschungsgruppe, die regional bedeutsame Untersuchungen über Nachhaltigkeitslücken durchführt. Zur Zeit arbeitet die Forschungsgruppe, unterstützt von Mitgliedern des Wissenschaftlichen Beirats, an einer Untersuchung »Regionale ökologische Berichterstattung für die Ulmer Region«.

\section{Vertrauen schaffen}

Strukturwandel bedeutet immer, daß es Gewinner und Verlierer gibt. Das gilt auch für nachhaltige Entwicklung. Entscheidend ist jedoch hierbei, daß Nachhaltigkeit nur in einer gemeinsamen Anstrengung aller Akteure erreicht werden kann. Dies ist eine entscheidende Kommunikationsaufgabe. Die Mitglieder des unw 
versuchen dieser Anforderung dadurch gerecht $\mathrm{zu}$ werden, indem sie nicht den anonymen brieflichen oder telefonischen, sondern den (zeitintensiven) persönlichen Kontakt im Gespräch suchen. In diesen Kontakten werden die positiven Leistungen einzelner Akteure herausgestellt. Es wird beispielhaft gezeigt, wer in welcher Weise durch Aktivitäten den Weg zur Nachhaltigkeit gegangen ist. Dies ist keine opportunistische Strategie, denn auch die »bitteren Wahrheiten « werden nicht verschwiegen

Der unw wird dem in seine Mitglieder gesetzten Vertrauen gerecht, indem er (wenige) Verąnstaltungen durchführt, die mit äußerster Sorgfalt vorbereitet sind und deren Thematik über den Tag hinaus reicht. Der unw präsentiert keine $\mathrm{Pa}-$ tentrezepte, sondern setzt auf das selbstorganisatorische Potential der einzelnen Akteure.

\section{Konkretes Alltagshandeln}

\section{durch Information verändern}

Der unw stellt Informationen und Rahmenbedingungen bereit. Rahmenbedingungen bereitstellen heißt im Wesentlichen, mit Hilfe von runden Tischen zu mediatisieren. Der erste runde Tisch fand im November als unw-Unternehmergespräch 1994 im Congress-Centrum Ulm statt und brachte 20 Ulmer Unternehmensleiter zusammen. Das Gespräch wurde von Ortwin Renn moderiert. Entscheidend war bestmögliche Qualität. Alle Veranstaltungen werden in Zusammenarbeit mit anderen Organisationen angeboten. Mit der Stadt Ulm führt der unw jedes Jahr eine große Veranstaltung im Ulmer Stadthaus durch. Die 1994er Veranstaltung im Mai behandelte das Thema »Ulms Zukunft sichern: Nachhaltige Wirtschaftsentwicklung«. Im Vortragsteil sprachen Repräsentanten von Stadt, Region und Land aus Wirtschaft, Wissenschaft und Politik. Am Podium nahmen wichtige Vertreter aus Unternehmen und Forschung teil, die zu konkreten Fragestellungen der nachhaltigen Entwicklung Stellung nahmen. Diese Veranstaltung hatte das Ziel, eine breite Öffentlichkeit zu informieren. Die 1995er Veranstaltung wird wiederum mit der Stadt Ulm zusammen durchgeführt und steht unter dem Motto »Wege zur Nachhaltigkeit« und findet am 18. Mai 95 statt.

Der unw informiert durch weitere Veranstaltungen im kleinen Kreis: runde Tische für die unterschiedlichen Akteure der Nachhaltigkeit, die Kirchen, die Wissenschaft, die Gewerkschaften, die Schulen, das Handwerk, etc. Beispielgebende Informationen sollen in Ausstellungen gegeben werden. Mit der Technologiefabrik (IHK) Ulm ist eine Ausstellung über zukunftsfähige Produkte geplant. Vortragsveranstaltungen für einzelne Akteure werden zur Zeit vorbereitet und im wesentlichen von Mitgliedern des Wissenschaftlichen Beirats bestritten. Eine Projektgruppe Energie beschäftigt sich mit der Einführung einer nachhaltigen Energieversorgung in Ulm.

Wichtige Informationsmedien sind die Schriftenreihe des unw und die unw-nachrichten.

\section{Informationen aus dem IÖW}

Personalia: Heike Gallert, wissenschaftliche Mitarbeiterin im Projektbüro Hannover, verläßt mit Abschluß des Projektes »Öko-Controlling in kleinen und mittleren Unternehmen in Schleswig-Holstein « das Institut. Zum 30. November hat Jutta Rentzsch ihre Arbeit im "Öko-Audit-Modellprojekt Märkisches Landbrot « abgeschlossen und ist seither als Mitarbeiterin in der BUND-Geschäftsstelle Bonn tätig. Birgit Soete verläßt zum Ende des Jahres das IÖW und arbeitet ab Januar 1995 als wissenschaftliche Mitarbeiterin an der FU-Berlin in Fachbereich Wirtschaftswissenschaften. Gösta Jancke wird zunächst für ein Jahr im IÖW an verschiedenen Projekten mitarbeiten. Er ist im Rahmen einer ABM-Stelle Mitarbeiter beim Forum für Arbeit und Projekte.

Projekte: Das Ökoforum, eine Kooperation mehrerer wissenschaftlicher Forschungsinstitute, hat vom Umweltbundesamt den Auftrag für ein Forschungsprojekt Stoffflüsse ausgewählter umweltrelevanter Stoffe bekommen. Das IÖW wird an diesem Projekt den ökonomischen Teil bearbeiten. Ansprechpartner sind Sabine Lehmann und Stefan Zundel.

Frieder Rubik und Kathrin Ankele bearbeiten als Unterauftragnehmer der Sozialforschungsstelle Dortmund die Evaluation der Möglichkeiten und ansatzweisen Durchführung der Methoden zur Interpretation und Bewertung der Sachbilanzen unter Beteiligung der Expertenarbeitskreise der Unternehmen, der Institute, Gewerkschaften und Beschäftigten. Eingebettet ist dieser Unterauftrag in das von der Deutschen Bundesstiftung Umwelt geförderte Projekt »Perspektiven für eine umwelt- und sozialverträgliche Werkstoffentwicklung in der Automobilindustrie unter Ein-

Als Band 1 der Schriftenreihe, die im Verlag »Wissenschaft \& Praxis« (Ludwigsburg/Berlin) erscheint, ist der Titel »Ökologisches Wirtschaften - Wege zur Nachhaltigkeit in Fallbeispielen « auf dem Weg, der seit Mitte November im Buchhandel vorliegt. In diesem Buch werden u. a. 40 Fälle von Unternehmen dargestellt, die durch Umweltschutzmaßnahmen Kosten eingespart oder neue Märkte erschlossen haben.

Der unw baut mehrere Datenbanken auf, die für vielfältige Informationen genutzt werden sollen. Die wichtigsten Datenbanken betreffen Beratung, Literatur und Adressen. Sie sollen bei Anfragen von Mitgliedern und Nicht-Mitgliedern herangezogen werden, um auf konkrete Informationen über Alltagshandeln zugreifen zu können.

\section{Forschung und Anwendung}

Der unw geht davon aus, daß reichhaltiges Wissen darüber vorliegt, wie Wege der Nachhaltig- beziehung von Zuliefer- und Verwerterunternehmen«, das im Oktober 1994 begann.

ÖKOFORUM: Vom 7. bis 9. Dezember fand in Rheine eine Tagung der Institute des ÖKOFORUMS in Kooperation mit dem Dialog Bildungswerk, Greven, statt. Das ÖKOFORUM wurde im Frühjahr 1990 gegründet und umfaßt folgende Institute: CONTRACT (Karlsruhe), IÖW, Institut für sozial-ökologische Forschung. (Frankfurt), Öko-Institut (Freiburg), Öko-Institut Südtirol (Bozen) und das Österreichische Ökologie-Institut (Wien). Titel der Tagung war Geld oder Leben? Ökologischer Konsum: Anspruchshaltung oder Genügsamkeit. Mit der Tagung sollte ein gemeinsames Leitbild für eine nachhaltige, ökologisch und sozial verträgliche Lebensweise entwickelt werden, ohne damit den Typus der einzelnen Institute zu nivellieren. Das Thema Ökologischer Konsum wurde als Dachthema ausgewählt, um ein solches Leitbild zu entwickeln. Eine Dokumentation der Ergebnisse ist für Anfang 1995 geplant. Die Veranstaltung wurde durch die IKEA-Stiftung, Hofheim unterstützt und richtete sich an die wissenschaftlichen Mitarbeiterinnen und Mitarbeiter der Institute.

Sonstiges: Frieder Rubik ist mit Wirkung vom 1. September zum Mitglied des Kuratoriums der Stiftung Warentest berufen worden.

Das IÖW-Berlin startet »wiedervereinigt $\ll$ in das neue Jahr: Ab Januar werden wieder alle Forschungsgruppen aus Berlin und die Verwaltung innerhalb eines Büros sein. Das vorübergehend in der Kantstraße bezogene Büro wird aufgegeben. Infolge des Umzugs und des technischen Fortschritts ändert sich ab Januar auch die Rufnummer für Berlin.

Die neue Nummer lautet dann:

$030 / 88459$ 4-0.

keit beschritten werden können. Gleichzeitig besteht jedoch ein großer Mangel an Wissen über die Umsetzung. Die wichtigste Zielsetzung des unw besteht daher darin, Informationen zu geben, die konkrete Handlungsänderungen anstoßen. Aus diesen Arbeiten ergeben sich spezifische Forschungsaufgaben, die dann wieder in die Anwendung geführt werden müssen.

\section{Bewertung}

Der Ulmer Initiativkreis ist nun ein gutes Jahr unterwegs. Welche Einsichten lassen sich daraus gewinnen?

- Das Ziel der Nachhaltigkeit ist klar: Wirtschaftsweisen und Lebensstile zu finden, die die sog. Regeln der Nachhaltigkeit aus der Knappheit von Quellen und Senken erfüllen. Um diese komplexe Aufgabe zu erfüllen, braucht es vieler Wege und vieler Initiativen mit unterschiedlichen Konzepten verschie- 
dener Gruppen. Diese Gruppen sollten konstruktiven Erfahrungsaustausch pflegen. Es gibt keinen Königsweg und kein Patentrezept. Die Ulmer haben damit begonnen, die Wirtschaft anzustoßen. Die Verwaltung wird folgen. Jedes benötigt seinen besonderen Weg.

- Gesellschaftliche Prozesse von innen her verändern zu wollen benötigt Zeit und Fingerspitzengefühl, und eine gesunde Streitkultur, um die unterschiedlichen Interessen kanalisieren zu können. Dafür muß zunächst Vertrauen geschaffen werden. Hier stehen wir in Ulm noch am Beginn.
- Der Umsetzungsprozeß ist pragmatisch, doch er muß von klaren theoretischen Vorstellungen geleitet sein. Diese zu entwickeln braucht Ruhe vor dem auffressenden Tagesgeschäft der vielen Termine. Geweckte Erwartungen wollen erfüllt sein und üben starken Druck aus. Trotzdem ist weniger oft mehr.

- Das Geld fließt nicht von alleine. Höchstmögliche Qualität an Veranstaltungen und Informationen zu liefern, kostet Geld.

Der Ulmer Initiativkreis nachhaltige Wirtschaftsentwicklung e. V. steht mit seinen regio- nalen Aktivitäten für mehr Nachhaltigkeit nicht allein. Weitere Beispiele sind Trier, Dessau und Leipzig. Diese Regionen müssen versuchen, ein Modell zu verwirklichen, das ansteckend wirkt auf andere. Warum soll nur Schnupfen ansteckend sein?

\section{Helge Majer, Stuttgart}

Ansätze für eine räumliche und ökonomische Integration

Die Flächennutzung im Kölner Stadtgebiet bestand 1991 u.a. aus 33\% bebauter Fläche (Haus- und Hofflächen, Betriebsgelände, Baustellen), 15,2\% Verkehrsfläche, 22,2\% landwirtschaftliche Fläche und 13,6\% Wald (1). Aufgrund altindustriell geprägter Strukturen und der verfolgten Wachstumsstrategie hat die Stadt Köln ein doppeltes Flächenproblem: einerseits in der Umnutzung und Aufwertung alter.Industriestandorte und andererseits im Rahmen ihrer Orientierung zum Dienstleistungsbereich die Deckung des wachsenden Bedarfs nach geeigneten Bürostandorten. Das 1989 erarbeitete Konzept für die Bereitstellung von Gewerbeflächen im Rahmen der Festlegungen des Flächennutzungsplanes sieht eine Angebotspolitik vor: Jährlich sollen ca. 15 ha Flächenreserve erschlossen und 15 ha Recyclingfläche für gewerbliche Nutzungen verfügbar gemacht werden. Dem steht ein Jahresverbrauch von 50 ha gegenüber, wobei zwischen einer Erweiterungsnachfrage für bestehende Standorte und Neuansiedlungen zu unterscheiden ist.

Der Druck zur Erschließung weiterer bebauungsreifer Reserveflächen von seiten der Wirtschaft im städtischen Umland läßt die Vorgaben der Landschaftsplanung zum Natur- und Landschaftsschutz als eine zu beseitigende Restriktion erscheinen. Die gewerbewirtschaftlichen Ansprüiche bedeuten damit einerseits die Auslagerung von Belastungen bzw. spezifischen Anforderungen aus der Stadt und andererseits die Bereitstellung wichtiger Versorgungsfaktoren wie Erholungsflächen oder Trinkwasser für die Stadt.

Der angerissene Problemkontext verdeutlicht folgende Konfliktfelder der Kölner Stadt-Umland-Beziehung:

- steigender Nutzungsdruck auf die umliegenden Flächen durch die städtische Gewerbepolitik,

- Veränderung der Standortanforderungen des sich neu ansiedelnden Gewerbes (»Gewerbe im Grünen «),

- Anstieg der Verkehrsströme und damit auch weitere Flächeninanspruchnahme, 
(c) 20I0 Authors; licensee IÖW and oekom verlag. This is an article distributed under the terms of the Creative Commons Attribution Non-Commercial No Derivates License (http://creativecommons.org/licenses/by-nc-nd/3.o/), which permits unrestricted use, distribution, and reproduction in any medium, provided the original work is properly cited. 